\title{
La cooperación científico-tecnológica-industrial para la defensa entre Argentina y Brasil
}

\author{
Defense scientific technological industrial \\ cooperation between Argentina and Brazil
}

Rev. Bra. Est. Def. v. 3, nº 2, jul./dez. 2016, p. 197-214

ISSN 2358-3932

\section{LEONARDO PABLO HEKIMIAN AURELIANO DA PONTE}

\section{DE QUÉ HABLAMOS CUANDO HABLAMOS DE INTEGRACIÓN}

La integración regional - en el marco de las relaciones internacionales contemporáneas - es un proceso por el cual dos o más Estados conforman una unidad política ${ }^{1}$ común ampliada, sin perder por ello su identidad original esencial, teniendo como objetivo la constitución de una comunidad con una finalidad propia y que represente al conjunto. Así, el proceso de integración está en construcción permanente, profundizándose cada vez más, pero al mismo tiempo deteniéndose siempre, por definición, antes de alcanzar un estadio tal que implicara la fusión total de sus partes integrantes en un nuevo todo soberano. Para ejemplificarlo claramente, ni Alemania ni Francia ni siquiera Luxemburgo han dejado de ser Estados nacionales soberanos por el hecho de integrar la Unión Europea y cumplir con los compromisos políticos, económicos y legales establecidos en el marco comunitario. Incluso en la eventualidad de incumplimiento de esos compromisos y posterior proceso de solución de crisis, como en el reciente caso griego, se advierte el mantenimiento de la esencia soberana de los Estados partes en difícil equilibrio con la pertenencia comunitaria.

En tal sentido, aunque un proceso de integración regional presente una metodología predominantemente económica, depende en su origen y configuración de una decisión política fundamental, impensable en un contexto de rivalidad geopolítica con hipótesis factible de solución militar. Por eso, en escenarios estratégicos signados por el riesgo de beligerancia, la

Leonardo Pablo Hekimian - Profesor Protitular de Política Exterior Argentina. Universidad Católica Argentina.

Aureliano da Ponte - Profesor da Escuela Superior de Guerra/Escuela de Guerra Naval. 
piedra fundamental para construir un esquema cooperativo radica en la superación del conflicto o al menos su canalización sincera y definitiva hacia procedimientos pacíficos de solución de controversias.

La secuencia de distensión - construcción de confianza - cooperación - integración, no es casual ni inédita en la historia contemporánea. El modelo más desarrollado de integración política y económica, la Unión Europea (aun con sus dificultades actuales), comenzó a gestarse una vez que dos grandes potencias europeas enfrentadas hasta el paroxismo en las dos guerras mundiales, Alemania y Francia, dieron por superadas sus históricas hipótesis de conflicto cruzadas. Prueba de ello fue que el primer acuerdo de integración que suscribieron, el de creación de la Comunidad Europea del Carbón y el Acero (Tratado de Paris, 1951), pactó la explotación conjunta de materiales críticos en la región centroeuropea. Es decir, que el objeto del acuerdo estaba constituido por uno de los elementos que en el pasado había generado las disputas internacionales que llevaron a la guerra. No lo hicieron por la repentina conversión a ideales altruistas, sino asumiendo de manera realista la inviabilidad de su futuro como naciones desarrolladas si no aunaban esfuerzos para enfrentar nuevos e inconmensurables desafíos comunes.

¿Qué sucedía en la región suramericana en los años setenta? Hablando de la relación argentino-brasileña, Massot (1998) utiliza una expresión que parece oportuna para definir las relaciones de Argentina no solo con el Brasil sino también con Chile y, con menor intensidad, con Paraguay y Uruguay: equilibrio de enemistades.

Sin embargo, hacia fines de los años setenta se advierte un significativo cambio de paradigma en el relacionamiento argentino con América Latina.

Siguiendo los estudios históricos de la Política Exterior Argentina (entre otros, Paradiso 1993; Russell 2010) se entiende que durante un periodo extenso de nuestra historia, más precisamente desde los albores de la organización constitucional de mediados de siglo XIX hasta las primeras décadas del XX, la Argentina desarrolló su Política Exterior de espaldas a la región circundante, a la que solo prestó atención prioritaria para solucionar conflictos territoriales o evitar una mayor injerencia de los Estados Unidos en aquellas primeras Conferencias Panamericanas. Pero ni la política ni el comercio ni las inversiones tenían en América Latina su foco atención, que básicamente estaba en Europa y más precisamente en Gran Bretaña. Los cambios profundos en el contexto mundial, así como el advenimiento de nuevas corrientes ideológicas y sociales a la esfera de decisiones políticas nacionales, implicaron también una evolución en los paradigmas del relacionamiento argentino con la región. 
A partir de esta consideración, pueden identificarse diversas etapas en el relacionamiento argentino con Brasil en particular y la región en general, siempre pensando en las políticas predominantes, no exentas de contradicciones en su ejecución histórica: ${ }^{2}$

I. Aislamiento regional: lo dicho, hasta la primera década del siglo XX.

II. Incipiente latinoamericanismo: podemos situar el inicio de esta etapa en las postrimerías del régimen conservador con el acuerdo $A B C$ entre Argentina, Brasil y Chile para la solución pacífica de sus controversias (1912) o a partir del advenimiento del gobierno de la Unión Cívica Radical con el presidente Hipólito Yrigoyen. Más político que económico, más retórico que de políticas concretas, significó un cambio de mirada hacia la región, que se fue acentuando - por necesidad - a medida que la crisis económica internacional golpeaba el modelo de inserción económica agroexportador. Se extiende hasta las postrimerías de la Segunda Guerra Mundial, abarcando así tanto a los gobiernos radicales, como los conservadores constitucionales y de facto que se sucedieron en los años treinta y cuarenta.

III. Latinoamericanismo activo: con la llegada de Juan Domingo Perón al poder, hay un cambio estratégico porque el fundador del Justicialismo concebía la unidad latinoamericana - inicialmente la subregional - como un factor esencial para desarrollar un nuevo modelo de inserción internacional. Lo interesante es que, aún con altibajos y en un contexto de inestabilidad institucional y deterioro político y económico, la tendencia a la afirmación de la integración regional tuvo hitos significativos más allá de 1955 y hasta fines de la década de 1960.

IV. Breve retorno al predominio de la rivalidad: los años setenta, por el contrario, estuvieron marcados por nuevas rencillas y el aumento de la tensión, incluyendo la planificación militar en torno de hipótesis de conflicto con los países vecinos. Las controversias con Brasil sobre la utilización de ríos compartidos y la construcción de represas, y sobre todo la tensión in crescendo con Chile en torno a la cuestión del Beagle, son ilustrativos de este periodo de enfriamiento de las relaciones regionales.

V. Neo-latinoamericanismo proactivo: desde la postrimería de aquella década del setenta, la clase dirigente argentina ha ido acentuando progresivamente, aún desde diversas perspectivas ideológicas, la idea de que la integración regional es un objetivo estratégico de la Política Exterior nacional. 
En efecto, el análisis histórico de una serie de hechos de las relaciones exteriores argentinas entre los años 1979 y 1982, muestra que aun antes del restablecimiento de las instituciones democráticas, la Argentina había comenzado a girar hacia América Latina, o al menos hacia sus vecinos subregionales. En 1979, el gobierno, a cargo de jefes militares pero nutridamente conformado por funcionarios civiles de alto nivel, especialmente en la Cancillería y el Ministerio de Economía, llegó a sendos acuerdos para la solución pacífica de controversias geopolíticas con Chile - acuerdo para someter a mediación el conflicto sobre el Canal de Beagle - y Brasil - por el aprovechamiento hidroeléctrico de las aguas del río Paraná-, y se había comprometido en un área de preferencias arancelarias con vistas a la futura conformación de un mercado común regional, mediante el ingreso a la Asociación Latinoamericana de Integración (Aladi 1980).

La solidaridad del Brasil y la mayoría de los países latinoamericanos por la Causa Malvinas tras el conflicto de 1982 - fuertemente contrastada con la indiferencia o directamente el claro alineamiento con Gran Bretaña de los Estados Unidos y los países de Europa Occidental que el régimen argentino había considerado países amigos -, terminó de generar un cambio de la cultura política de la dirigencia argentina, que quizás por primera vez en décadas asumió su condición de país periférico y latinoamericano.

La recuperación de la democracia vino a consolidar este incipiente proceso. Sumados al Tratado de Paz y Amistad con Chile (1984), Raúl Alfonsín inició y Carlos Menem consolidó dos procesos muy significativos para la integración con Brasil: el de la superación de la desconfianza vinculada a la capacidad de utilización de la tecnología nuclear, y el de la cooperación y complementación política y económica. Expresiones de estos procesos fueron, entre otros, la Declaración de Iguazú (1985), el establecimiento del Programa de Integración y Cooperación Económica entre la Argentina y el Brasil (1986), la conformación del Mercosur junto a Paraguay y Uruguay (1991) y los sucesivos acuerdos para garantizar el uso pacífico de la energía nuclear, suscritos entre 1985 (aunque con antecedentes ya en 1980) y 1991. Como se advierte, si bien en los años noventa el gobierno de Menem adoptó un modelo de política exterior totalmente diferente al de su predecesor, en ambos periodos hubo cabida y un lugar destacado para la integración regional.

Los primeros años del siglo XXI, pese a que se abrieron con una crisis política y económica sin precedentes, lejos de revertir el objetivo de integración regional, han procurado revalorarlo - cierto que con éxito relativo y desparejo -, llegando a la activa participación argentina en la conformación, por primera vez, de un bloque político subregional, como lo es la Unión de Naciones Suramericanas (Unasur), promovido por Brasil. 
Los últimos años han reforzado la idea de asociación estratégica entre Argentina y Brasil. Así, el 31 de enero de 2011, las máximas autoridades suscribieron una Declaración Conjunta, en la que sostuvieron conceptos tales como:

- Reafirmaron la importancia de la relación estratégica entre la Argentina y Brasil como eje constitutivo de la integración a nivel regional y reiteraron su compromiso con el proceso de integración bilateral como una política de estado en ambos países.

- Consideraron a la alianza estratégica como piedra fundamental para el éxito del proyecto común de integración, en el cual destacan la importancia de la profundización del Mercosur como principal ámbito de integración política, social, económica y comercial de la región, y la constitución de la Unasur como factor de unidad y de cooperación en América del Sur.

- Ratificaron la vigencia del Mecanismo de Integración y Coordinación Bilateral y determinaron la atención prioritaria al desarrollo de nuevos proyectos que consoliden una vigorosa agenda de cooperación en el área social, más allá de la aceleración e intensificación de los proyectos existentes en las áreas estratégicas de cooperación nuclear, espacial, defensa, industria naval, industria aeronáutica, infraestructura, energía, y digital.

En tono con este marco político, en abril de 2013, los ministros de Defensa firmaron la Declaración de Río de Janeiro, en la que reiteraron la vitalidad de la Asociación Estratégica Argentina-Brasil en el campo de la defensa y reafirmaron su compromiso con el continuo fortalecimiento y con la dinámica de la cooperación bilateral, tanto en términos de la política de defensa cuanto de la política industrial para el sector. Esta misma línea discursiva se mantiene hasta el presente, con la relevante novedad que ya se ha verbalizado en un instrumento bilateral suscrito por los nuevos gobiernos surgidos en ambos países entre fines de $2015 \mathrm{y}$ comienzos de 2016. En efecto, el 23 de mayo de 2016, en su primera visita a la Argentina tras ser designado canciller del gobierno de transición brasileño, José Serra suscribió, con su par argentina Susana Malcorra, un Memorando de Entendimiento intergubernamental para el establecimiento de un Mecanismo de Coordinación Política. El referido instrumento se encuadra "dentro del marco de los festejos por los treinta años de la suscripción de la Declaración de Foz de Iguazú, el 30 de noviembre de 1985, que marcó el inicio del proceso de aproximación entre Argentina y Brasil y resultó en la construcción de una relación caracterizada por la confianza mutua, la amistad y la cooperación, en nombre de los ideales 
de paz, libertad y justicia social; y por los veinticinco años de la creación, el 26 de marzo de 1991, del Mercosur, el proyecto de integración más ambicioso de la región [...]". Asimismo enfatiza "la importancia de los vínculos políticos entre Argentina y Brasil, cuya alianza ha resultado determinante para la promoción del desarrollo de ambos países y la profundización del proceso de integración regional, llevando a la construcción de una América del Sur más integrada, pacífica y próspera” y expresa que el mecanismo que se crea "tendrá como principales objetivos el intercambio de opiniones sobre temas de las agendas bilateral, regional y global, con el fin de coordinar posiciones; y el seguimiento de proyectos estratégicos de integración bilateral, especialmente en las áreas de la ciencia, la tecnología y la innovación; la defensa; la industria aeronáutica; la energía; y el comercio" (art. 2 - énfasises nuestro).

A tenor de estas expresiones conjuntas documentadas y otras muchas que se remontan al menos a $1985,{ }^{3}$ no parecería haber duda de que existe una asociación estratégica argentino-brasileña, sucesivamente proclamada. Más allá de los avatares políticos y económicos que cada país ha experimentado en las últimas décadas, existen numerosos indicadores que evidencian vínculos notables, sea en el terreno político, como en el económico, social e incluso en el terreno de la defensa y la seguridad. No obstante, un análisis profundo da cuenta de un proceso en construcción que requiere ajustes cualitativos para desarrollarse plenamente.

\section{BALANCE ESTRATÉGICO DE 30 AÑOS DE INTEGRACIÓN}

Tres décadas después de iniciado, el proceso de integración argentino-brasileño y su proyección regional, puede ser criticado por incompleto, desparejo e institucionalmente débil. Sin embargo, no hay opción política viable que lo rechace, ni modelo económico que no lo contemple ni planeamiento militar que no lo incluya como una fortaleza propia, lo cual es de significación estratégica.

No obstante, dice Morgenthau (1986) que una alianza puede calificarse como operativa cuando "[...] sea capaz de coordinar las políticas generales con las medidas concretas correspondientes a cada uno de sus miembros [...] [para lo cual] es preciso que estos integrantes estén de acuerdo no sólo con los objetivos generales sino también con políticas y medidas concretas." Evidentemente, existe un riesgo considerable de que los objetivos propuestos en los sucesivos jalones del proceso de integración argentino-brasileño tengan dificultades para derivar en políticas y acciones concretas, paso indispensables para convertirse en un esquema de concertación realmente operativo. Más, aun esta incertidumbre estratégica se extiende a 
todo el proceso de integración regional, que requiere de la vitalidad de la asociación argentino-brasileña para su consolidación.

Hablando concretamente del ámbito de la Defensa, la consolidación de este proceso y su eventual profundización, con vistas a la salvaguarda de intereses regionales - construidos sobre la base de los factores comunes, compatibles y/o complementarios de los intereses nacionales - presenta requerimientos que implican un mayor compromiso de los Estados partes hasta alcanzar niveles de verdadera integración de los sistemas de defensa nacionales.

En cuanto a las posibilidades de impulsar proyectos científico-tecnológicos o productivos, particularmente relevante resulta el análisis del caso del avión CBA-123 (Cooperación Brasileño Argentina). A partir de los entendimientos iniciados con la firma del Acta de Foz de Iguazú, el 17 de enero de 1986 se refrendó un acuerdo de cooperación técnico industrial entre el Ministerio de Defensa argentino (a través del Área Material Córdoba) y Embraer. Con una vigencia de quince años reajustables cada tres de común acuerdo en función de su evolución, ambas empresas podían proyectar, desarrollar, ensayar y fabricar aeronaves nuevas, equipos, sistemas y partes para uso civil y militar. En julio de ese mismo año, como parte del Programa de Integración y Cooperación Económica (Pice) se incluyó un instrumento legal llamado Protocolo 12 de Cooperación Aeronáutica que estableció los siguientes propósitos: a) Integración en el sector aeronáutico para el desarrollo y fabricación de aviones en forma conjunta; b) Desarrollo, producción y comercialización de aeronaves, cubriendo los acuerdos existentes; e integrar sectores que posean tecnología de punta. Sin embargo, tan plausibles objetivos no fueron logrados en los años siguientes. Al tiempo que la coyuntura económica fue afectando en forma creciente los compromisos asumidos, las diferentes concepciones y estrategias que orientaron a los sectores industriales de defensa también explican por qué quedó trunca esta iniciativa.

En los años recientes, los acuerdos y proyectos definidos en el marco bilateral y del Consejo de Defensa Suramericano apuntan a este tipo de fines, pero para concretarse requieren un empeño de recursos y una adaptación de doctrinas y capacidades propias que aún se halla pendiente. Ése es el desafío principal que tienen ambos países para el próximo lustro. El estado de situación de los acuerdos y mecanismos acordados en materia de defensa entre Argentina y Brasil en particular y en el marco de Unasur, apuntan a objetivos que superan los instrumentos típicos de un esquema de defensa cooperativa, limitados a la generación de confianza, la prevención de conflictos y medidas de cooperación e intercambio que podríamos llamar de primera generación. Puede considerarse entonces 
que podríamos aspirar al establecimiento de un sistema que podría denominarse de Defensa Integrada, conformado por instituciones, mecanismos, políticas y normas consensuadas, que parten de una visión estratégica de intereses regionales.

Para comenzar a trabajar en ello, es necesario avanzar hacia la definición de planes, programas y proyectos comunes, con metas de ejecución. No obstante, es menester seleccionar con prudencia las áreas estratégicas para progresar en la integración defensiva. Es decir, pensamos que más que un plan omnicomprensivo, tiene mayores posibilidades de éxito asumir compromisos concretos en campos de acción considerados propicios para promover políticas comunes. En tal sentido, nos permitimos proponer los siguientes:

- Estrategia de protección de recursos: Argentina, Brasil y prácticamente la totalidad de los miembros de Unasur tienen una visión similar en esta materia, como quedó reflejado en la Declaración final de la reunión del CDS de 2011, donde se resaltó la importancia de que el Consejo de Defensa Suramericano incluya en el plan de acción de 2012 proyectos vinculados a la protección de la biodiversidad y los recursos naturales. Paralelamente, se recomendó al Centro de Estudios Estratégicos que inicie un estudio sobre los riesgos y las amenazas en este campo. Y se ha creado un Grupo de Trabajo sobre Defensa y Recursos Naturales para avanzar en la elaboración del inventario de los recursos naturales estratégicos de la región en coordinación con el CEED-CDS. Del trabajo conjunto y efectivo que encaren Argentina y Brasil depende en buena medida que esta incipiente preocupación regional derive en la elaboración de una verdadera doctrina en la materia para las Fuerzas Armadas de la región.

- Posturas comunes y coordinadas en el escenario internacional: Cualquiera que haya participado de foros hemisféricos o internacionales sobre temas de defensa y seguridad internacional, sabe que son muchas las ocasiones en que los países suramericanos se encuentran del mismo lado de la mesa cuando se tratan asuntos controversiales, en los que potencias o bloques extra-regionales tienen posiciones previamente acordadas y suelen actuar mancomunadamente, incluso influyendo sobre países que arriban con posturas indefinidas. Sin embargo, esta natural confluencia de intereses entre los países latinoamericanos muchas veces no puede expresarse con toda la fuerza que debería, por carecer justamente de instancias de coordinación previa, necesarias para ajustar posiciones, limar matices y encarar modos de acción común. Los mecanismos bilaterales de diálogo en Defensa tienen un importante potencial en este sentido. 
- Emprendimientos comunes en materia de ciencia, tecnología e industria de defensa: en esta materia, sensible como ninguna otra, la conformación de equipos de trabajo científicos y tecnológicos bilaterales, así como la constitución y ejecución de acuerdos industriales empresarios e interestatales, constituyen pasos fundamentales para la verdadera integración en defensa. En la segunda parte de esta ponencia, nos dedicaremos a profundizar esta idea.

\section{INDUSTRIA Y TECNOLOGÍA DE DEFENSA: CONSIDERACIONES SOBRE SU DOBLE CARÁCTER}

Antes de señalar consideraciones sobre los denominadores comunes vis a vis los contrastes entre Argentina y Brasil, es pertinente apuntar algunas reflexiones sobre la naturaleza de la Industria y Tecnología de Defensa (en adelante, ITD). Primero, entraña una complejidad particular no siempre contemplada por los responsables de formular un Programa de cooperación. El tipo de bien o servicio y sus implicancias en términos de poder en el sistema internacional, así como las dificultades emergentes propias de una actividad industrial con cierta intensidad tecnológica, son características definitorias. El problema es que lejos de consistir en una cuestión teórica, la falta de dimensionamiento de estos aspectos incide negativamente en el momento de la ejecución. Segundo, cualquier emprendimiento o proyecto exige como punto de partida comprender su doble carácter, político-estratégico y económico-industrial.

En el plano político-estratégico, la decisión de cooperar implica cierto grado de acuerdo respecto a la apreciación del entorno estratégico y, dentro de ese marco, la identificación de riesgos y amenazas compartidas que requieren capacidades militares adecuadas para ser repelidas. Por lo tanto, deben recibir el respaldo de las máximas autoridades pero, sin embargo, esta condición es necesaria y básica pero no suficiente ya que es fundamental el diseño de instrumentos que acompañen y den concreción al proceso. En el plano económico-industrial es central articular las dimensiones educación-ciencia con tecnología-producción. Generar herramientas que contribuyan a la densificación de las cadenas productivas y a su interconexión. Recursos para el financiamiento y una escala de producción industrial mínima rentable. Este último es un aspecto crítico sobre todo en economías emergentes a fin de poder competir contra las condiciones de precio y calidad de la industria extranjera. Asimismo, se requieren procesos dinámicos de producción orientados por las lógicas diferenciales de cada sector específico. Finalmente, una sofisticada comercialización que involucre al Estado así como mecanismos de fomento y promoción. Además, 
los diagnósticos son muy importantes para perfilar las políticas públicas. Por eso, analizar el mercado de armas y el segmento dentro del mismo correspondiente a un producto o servicio, conocer a los actores nacionales e internacionales y sus diferentes intereses, así como quiénes son los competidores y quiénes son los clientes potenciales.

\section{CONVERGENCIAS ESTRUCTURALES Y ESTRATEGIAS DIVERGENTES}

Una revisión global de antecedentes de los dos países analizados respecto al desarrollo de industrias y sectores vinculados a la Defensa nacional permite un ejercicio comparativo que, en el contexto latinoamericano, los ubica a ambos en el mismo cuadrante. Dicho de otro modo, es posible identificar procesos macro que tienen puntos en común durante el siglo $\mathrm{XX}$ que incluso se reeditan en el presente. En lo general, esquemas económicos primario-exportadores hasta 1930; industrialización por sustitución de importaciones con activa participación estatal; neoliberalismo y desestatización de la economía. En lo particular, impacto de la Primera Guerra Mundial sobre el pensamiento estratégico de algunos sectores políticos, económicos y militares que derivó en el surgimiento de una corriente favorable al desarrollo industrial nacional y consolidación de las Fuerzas Armadas como actor relevante.

Ahora bien, cuando se avanza en el estudio a nivel país se advierten patrones o esquemas de industrialización heterogéneos que se reflejan en las trayectorias particulares. Es decir, Argentina y Brasil han compartido ciertas similitudes en los fundamentos explicitados en sus decisiones de impulsar el desarrollo de sus industrias de defensa. Esta afirmación se refiere solamente a ciertos objetivos generales que se enuncian pero no a las acciones que se derivan de ellos para alcanzarlos (Abetti y Maldifassi 1994). Franko (1996) señala que durante el siglo pasado, tanto las estructuras industriales de defensa de Argentina, Brasil y Chile como sus itinerarios posteriores evidencian un patrón diferente (respectivamente ambiciosa promoción estatal, asociación público-privada, minimalista con orientación externa), y afirma que la industrialización en el sector se ha correspondido con el modelo de desarrollo de cada economía.

Un estudio previo concluye que el suceso de las firmas brasileñas en términos de su participación en los mercados internacionales en los años ochenta responde a la estructura que adquirió la industria y la forma en la cual se organizó, destacándose el relacionamiento entre el Estado y el sector privado así como la atención dispensada a las señales del mercado (Franko-Jones 1992). En cambio, una visión que concibió que el desarrollo nacional solo podía realizarse exclusivamente a través de lo estatal, sumado 
a la inestabilidad institucional argentina, impactaron negativamente sobre la industria de defensa (Da Ponte 2010).

Sea como fuere, estas culturas estratégicas influyeron en mayor o menor medida en los distintos intentos de cooperación bilateral tal como fue ejemplificado. Por eso es clave advertirlas para comprender con precisión los resultados del pasado, los factores favorables y las dificultades.

\section{PROGRAMAS DE COOPERACIÓN EN INDUSTRIA Y TECNOLOGÍA DE DEFENSA: GENERALIDADES}

La participación en un proceso de cooperación debe representar ganancias efectivas y perceptibles para los actores participantes. Evidentemente, la interacción entre dos actores es muy compleja, por lo que la falta de planificación que integre las múltiples variables intervinientes, a la vez que considere los instrumentos institucionales más convenientes, atenta decisivamente contra las posibilidades reales de obtener resultados positivos. Los Programas de Cooperación en ITD constituyen una unidad analítica que permite reflexionar desde aspectos tan amplios como la concepción estratégica-militar, los presupuestos y las capacidades en I\&D y productivas, hasta asuntos legales y técnicos (certificaciones, homologaciones, normalizaciones, etc.). En otras palabras, posibilita indagar en los múltiples niveles de un proceso cooperativo. Esquemáticamente, se componen de los siguientes elementos:

\section{ACUERDOS E INSTRUMENTOS INSTITUCIONALES}

En primer lugar, es una condición sine qua non que se generen acuerdos que sean acompañados por instrumentos institucionales efectivos porque, de lo contrario, no es posible concretar las iniciativas. En segundo lugar, pueden distinguirse tres niveles de actores. En el máximo nivel están los decisores políticos y, en aquellos casos donde hay empresas de defensa (pública y/o privada), los gerentes de las mismas. Luego, los directores de los programas y los equipos de trabajo. Tercero, los potenciales usuarios. Esta distinción es relevante para comprender que son necesarios entendimientos e interrelaciones en cada uno de estos niveles. Es decir, no alcanza con que se alcancen arreglos formales de tipo político si esto no se traduce en las instancias inferiores. En tercer lugar, un programa de esta naturaleza no finaliza cuando un sistema o equipo es incorporado al instrumento militar del comprador, ya sea de los países involucrados o de un tercero en caso de que eventualmente sea exportado, sino que debe continuar hasta el final de su ciclo de vida útil (en el mantenimiento, el ciclo logístico, etc.). 
Es importante el impulso de algunas medidas como por ejemplo el aumento de intercambio de información sobre programas de I\&D, presupuestos plurianuales para los programas que se aprueben (garantizando inversiones de largo plazo), una arquitectura legal adecuada y mecanismos que incentiven la realización de actividades combinadas de I\&D, la innovación empresarial y la protección de nichos tecnológicos.

En un nivel más específico directamente relacionado con el diseño y concepción de un producto, Ferreira da Silva (2010) propone la elaboración de criterios amplios, es decir, con arreglo a ciertas capacidades operacionales en lugar de consideraciones meramente técnicas, debido a que este tipo de definiciones suelen llevar más tiempo para arribar a consensos. Esto busca facilitar las cosas debido a que existe el peligro de que una excesiva sofisticación y diversidad de demandas al momento de la planificación derive en un sistema caro y complejo que obstaculice su materialización. ¿Por qué resulta apropiado plantear la cuestión de esta manera? Porque este razonamiento procura integrar los elementos político-estratégicos con los económico-financieros. Al establecerse parámetros técnicos mínimos, es decir, al trabajar en un esquema modular de forma que permita consensuar un producto cuyas especificidades genéricas constituyan un modelo básico que sea competitivo (en términos operacionales y económicos), aquellos requerimientos tecnológicos y logísticos particulares que excedan ese estándar, pueden incorporarse según la configuración que prefiera el cliente previéndose diversas variantes. Lógicamente hay subsistemas y componentes que necesariamente deben ser comunes ya que son determinantes para el diseño, pero hay otros que no lo son. En todo caso, esto puede ser aprovechado en forma inteligente ganando el producto en versatilidad y flexibilidad o ser perjudicial demorando el programa.

\section{EDUCACIÓN PARA LA DEFENSA: FORMACIÓN Y ADIESTRAMIENTO PARA LA COOPERACIÓN}

Sintéticamente, la educación en el ámbito de la defensa articula tres universos: enseñanza, entrenamiento y formación (Kalil Mathias y Berdu 2013). Por cierto, a estos podría agregarse la capacitación y el perfeccionamiento. En este contexto, la formación y el adiestramiento para la cooperación de civiles y militares de acuerdo a sus roles constituye un área esencial para fortalecer la convergencia de intereses en el mediano y largo plazo. En efecto, la generación y articulación de actividades que aborden la cooperación industrial y tecnológica como problemática parece una herramienta clave en este sentido. En definitiva, es fundamental que se la considere una dimensión estratégica cuando se concibe un programa de 
estas características no sólo porque es sustancial en su formulación sino también en su ejecución.

Justamente su importancia se comprende más cabalmente a través de algunas de sus derivaciones prácticas. Por mencionar una, en la concepción de un Sistema de Armas subyacen elementos doctrinarios, el concepto de empleo, entre otros. Este punto tiene estrecha relación con los distintos momentos de su formación y consecuente adiestramiento. Por ello, la formación debe, en principio, dialogar con el diseño conceptual y los requerimientos operacionales, aunque lo ideal es que forme parte desde las etapas iniciales de planeamiento del proyecto. Las cuestiones educativas y tecnológicas deben estar profundamente conectadas en un programa de ITD.

\section{GESTIÓN Y EQUIPOS INTEGRADOS DE TRABAJO}

En perspectiva comparada, los programas de cooperación que han alcanzado cierto éxito hasta el momento se han caracterizado por la composición multinacional de sus equipos gerenciales y de trabajo. Esto que parece obvio, sin embargo, no siempre es posible. Como afirma Ferreira da Silva (2010), "cuanto más integrado es un equipo, menos poder tendrán los ministerios de defensa sobre esos programas”. Es pertinente aclarar que el autor no se refiere a desvincular el programa de los lineamientos políticos. Por el contrario, lo que supone es que los Equipos Integrados de Trabajo (EIT), tal como son denominados, han demostrado un salto cualitativo en cuanto a que sus participantes pasaron de actuar simplemente como representantes nacionales (sujetos a lógicas de corto plazo), a conformar un grupo enfocado en alcanzar satisfactoriamente los objetivos planificados. Asimismo, partiendo de la base del tipo de producto y de que las Fuerzas Armadas de los países involucrados serán en muchos casos los principales usuarios y, en otros, los únicos, es adecuado que asesoren en las diferentes fases de un programa.

\section{FOROS Y REDES DE COOPERACIÓN MULTINIVEL}

El funcionamiento de un programa depende en gran medida del conocimiento entre los actores que participan. De acuerdo a la experiencia europea, la interacción entre políticos, funcionarios estatales, diplomáticos, militares, empresarios y académicos con la finalidad de fomentar el entendimiento mutuo ha influido directamente y de manera positiva en el gerenciamiento y la ejecución de los programas (Darnis et al. 2007). Al conocer cómo piensa cada actor y cómo resuelve un problema concreto, se producen condiciones para el establecimiento de criterios compartidos. Las 
comunicaciones "multinivel" entre los actores estatales y no estatales tienen un efecto significativo positivo para los programas en ITD. Espacios como foros empresarios, redes académicas, prensa especializada, observatorios tecnológicos, Congresos, Seminarios y Workshops en materia de cooperación en ITD de acuerdo a los niveles que han sido diferenciados previamente son modalidades a explorar.

\section{CONSIDERACIONES FINALES}

En la actualidad, Argentina y Brasil, al igual que sus pares suramericanos, sostienen una postura estratégica de carácter defensivo, conforme la cual la concepción y la disposición estratégica, la política de defensa y su consecuente política militar, el diseño de fuerzas y la previsión de empleo del instrumento militar, se encuentran estructurados según el principio de legítima defensa ante agresiones de terceros estados.

Por otro lado, en un mundo interrelacionado e interdependiente, las naciones de desarrollo intermedio tienden a concebir su defensa en la doble dimensión "autónoma” por un lado, y "cooperativa” por otro. Los sistemas de defensa nacionales, su diseño de fuerzas y sus capacidades, se vinculan y complementan con los compromisos multilaterales, especialmente de nivel regional.

La constitución del CDS en el marco de la UNASUR, si realmente aspira a alcanzar sus objetivos, conlleva el compromiso y el esfuerzo de los Estados miembros en pos de una coordinación cada vez más estrecha entre sus sistemas de defensa y sus fuerzas armadas. Para ello, debería primar el convencimiento de que, ante la incertidumbre estratégica global y los vastos recursos regionales a defender, no hay posibilidades de plantear una defensa nacional efectiva sin concebirla en el marco de la cooperación y la complementación regional, en función de los intereses comunes de los países miembros.

No se trata de plantear un proceso de integración en defensa de carácter meramente idealista o retórico, no obstante que los valores humanos que nos aúnan son de por sí fundamento esencial para avanzar en este camino. Más allá de ello, se plantea la profundización de la cooperación regional en materia de defensa partiendo de una postura realista, es decir, sobre la base de los factores comunes de nuestros intereses nacionales estratégicos que, como se dijo, alcanzan el grado de interés regional. El desarrollo autónomo no es incompatible con la cooperación sino que puede, como resultado de impulsos efectivos, ser complementaria. En todo caso, el asunto pasa por comprender que la dimensión cooperativa se apoya, y debe ser funcional, a los objetivos propios. Los estudios revelan diversas motiva- 
ciones para la cooperación industrial en defensa en el caso de los países de Europa, que abarcan desde la disminución de costos de I\&D y producción y/o la generación de economías de escala por aumento de demanda, hasta el desarrollo de equipamiento común que favorezca la interoperabilidad, entre otros (ISS 2008). Se observan razones estratégicas, geopolíticas y económicas.

En tal sentido, no se puede consolidar la alianza estratégica bilateral, esencial para el desarrollo positivo de la integración regional, si no se basa en proyectos concretos y de envergadura en áreas de cooperación relevantes para el desarrollo nacional, siendo la científico-tecnológico-industrial en el ámbito de defensa una de las de mayor potencial. Para ello resulta relevante comprender profundamente las características y los condicionantes que constituyen su naturaleza que inevitablemente es compleja. La cooperación bilateral representa una oportunidad potencial vis a vis un doble desafío. Es central sintonizar frecuencias y concepciones respecto de las estrategias y modelos de desarrollo sectorial. La edificación de estos cimientos implica generar acciones para los cuatro elementos involucrados en un Programa de Cooperación en ITD. Solo en la medida en que se atienda la multidimensionalidad intrínseca de proyectos de este tipo y se dediquen esfuerzos concretos será posible avanzar con experiencias exitosas que consoliden la alianza estratégica bilateral.

\section{REFERENCIAS}

Abetti, P., Maldifassi, J. 1994. Defense Industries in Latin American Countries: Argentina, Brazil, and Chile. London: Praeger.

Darnis, J.-P., Gasparini, G., Grams, C., Keohane, D., Liberti, F., Maulny, J.-P., Stumbaum, M.-B. 2007. Lessons learned from European Defence equipment programmes. Ocassional paper, 69. European Union: Institute for Security Studies.

Da Ponte, A. 2010. Desarrollo tecnológico e industrias para la defensa en Argentina: aportes para el debate. Cuadernos de Actualidad en Defensa y Estrategia, Buenos Aires, 5, 33-56.

Ferreira Da Silva, P. 2010. Aprendendo a cooperar: a experiência recente da união européia em programas conjuntos de produtos de defesa. In: Munhoz Svartam, E., Arias Neto, J. M., Pires de Godoy, T. R., Alves, V. C. (Org.). Defesa, Segurança Internacional y Forcas Armadas. Campinas: Mercado de Letras.

Franko, P. 1996. Defense production in Argentina, Brazil and Chile: A comparative perspective. Defense Analysis, 12 (3), 315-326. 
Franko-Jones, P. 1992. The Brazilian defense industry. Colorado: Westview Press.

Hekimian, L. 2016. Estructura y Actualidad de la Política Exterior Argentina. Revista Iberoamérica, Moscú, 4, 33-53.

Kalil Mathias, S., Berdu, G. P. 2013. Ensino Militar no contex to da mercantilização da educação. In: Alves do Carmo, C., Winand, É. C. A., Barnabé, I. R., Miranda Pinheiro, L. (Org.). Relações internacionais: Olhares cruzados. Brasília: Fundação Alexandre de Gusmão.

Lanús, J. A. 1984. De Chapultepec al Beagle. Buenos Aires: Emecé.

Massot, V. G. 1998. Del equilibrio de enemistades a la cooperación bilateral. In: De la Balze, F. (Comp.). Argentina y Brasil enfrentando el siglo XXI. Buenos Aires: CARI.

Morgenthau, H. J. 1986. Política entre las naciones. La lucha por el poder y la paz. Buenos Aires: Grupo Editor Latinoamericano.

Paradiso, J. 1993. Debates y trayectoria de la política exterior argentina. Buenos Aires: Grupo Editor Latinoamericano.

Puig, J. C. 1975. La Política Exterior Argentina y sus tendencias profundas. Revista Argentina de Relaciones Internacionales, Buenos Aires, I (1).

Russell, R. 2010. La Argentina del segundo centenario: ficciones y realidades de la Política Exterior. In: . Argentina 1910-2010. Buenos Aires: Taurus. 


\section{NOTAS}

1. La expresión en idioma inglés "polity" sería muy apropiada en este caso.

2. Lo que sigue es un esquema de elaboración propia, que el artículo se desarrolla sintéticamente sobre la base de los análisis históricos de la Política Exterior Argentina de varios autores.

3. Sería el caso de los documentos oficiales de reseña de su política exterior que elaboran ambas Cancillerías como, por ejemplo, los objetivos estratégicos de la Secretaría de Relaciones Exteriores de la Argentina, publicados en el sitio de Internet de la Cancillería, que hasta 2010 expresamente incluían "profundizar la alianza estratégica con Brasil”. La utilización de una fórmula más genérica a partir de 2011 ("profundizar la integración política con los países de América Latina”) no debería leerse como un cambio de política sino como una expresión diplomática destinada a evitar el descontento de otros países de la región. 


\section{LA COOPERACION CIENTIFICO-TECNOLOGICA-INDUSTRIAL PARA LA DEFENSA ENTRE ARGENTINA Y BRASIL}

\section{RESUMEN}

El artículo reseña la evolución del vínculo bilateral, enfocando en las oportunidades y desafíos para una asociación estratégica, con el fin de destacar el rol potencial que la cooperación científico-tecnológica-industrial en Defensa (CTID) ocupa. El análisis incluye los contrastes y convergencias entre los modelos de desarrollo industrial en defensa y los antecedentes y perspectivas de la CTID bilateral.

Palabras claves: Asociación Estratégica; Argentina; Brasil; Cooperación Científica Tecnológica Industrial; Seguridad Internacional.

\section{ABSTRACT}

The article outlines the evolution of the bilateral link, focusing on the opportunities and challenges for a strategic partnership, in order to highlight the potential role that scientific-technological-industrial cooperation in Defense (CTID) occupies. The analysis includes the contrasts and convergences between the models of industrial development in defense and the antecedents and perspectives of the bilateral CTID.

Keywords: Strategic Partnership; Argentina; Brazil; Industrial Technological Scientific Cooperation; International Security. 